\title{
Lutter contre le dopage par une dépénalisation reconstructive du sportif coupable : les enjeux et les ambiguïtés du projet Windop ${ }^{\star}$
}

\author{
Alexandra Veuthey ${ }^{1}$, Christophe Jaccoud ${ }^{2}$, Dominique Malatesta ${ }^{3}$ et Yann Hafner ${ }^{4}$ \\ 1 Faculté de droit, Université de Melbourne, 185 Pelham Street, 3053 Carlton, Victoria, Australie \\ 2 CIES-Université de Neuchâtel, Avenue DuPeyrou 1, 2000 Neuchâtel, Suisse \\ 3 HES SO Travail social et Santé, EESP, 14, ch. des Abeilles, 1010 Lausanne, Suisse \\ 4 Faculté de droit, Université de Neuchâtel, Av. du 1er Mars 26, 2000 Neuchâtel, Suisse
}

Reçu le 14 février 2015 - Accepté le 18 mars 2015

\begin{abstract}
Résumé. La lutte contre le dopage montre une tendance croissante à articuler un axe du droit, construit sur la sanction, et un axe de la régulation enraciné dans des principes d'arbitrage et de conciliation attentifs aux notions de dignité des sportifs. Nous illustrons notre propos et les ambiguïtés de cette lutte à travers l'analyse socio-juridique des premiers éléments de mise en œuvre du projet-pilote de lutte antidopage Windop. Ce dernier, soutenu financièrement par l'Agence mondiale antidopage (AMA) et bénéficiant de l'expertise de sociologues et de psychologues, vise depuis 2010 à réhabiliter des sportifs convaincus de dopage, à travers diverses mesures d'accompagnement destinées à reconnaître la faute, à prévenir les récidives et à reconstruire un avenir sportif.
\end{abstract}

Mots clés : Dopage, civilisation du droit, Tribunal arbitral du sport, Code mondial antidopage, windop, plea bargaining

\begin{abstract}
Fighting against doping by a reconstructive decriminalization of the guilty athlete: challenges and ambiguities of the Windop project.

The fight against doping shows an increasing trend towards articulating an axis of law, built on sanctions, and an axis of regulation rooted in arbitration and conciliation principles mindful of the notions of athletes' dignity. We illustrate our argument and the ambiguities of this fight through a socio-legal analysis of the first elements of the implementation of the anti-doping pilot project Windop. This project, financially supported by the World Anti-Doping Agency (WADA) and benefitting from the expertise of sociologists and psychologists, has, since 2010, intended to rehabilitate athletes found guilty of doping, through various support measures aimed at recognizing the fault, preventing repetition of an offence and rebuilding a sporting future.
\end{abstract}

Key words: Doping, civilization of law, Court of arbitration for sport, World anti-doping code, Windop, plea bargaining

\section{Introduction}

Si la volonté de punir les sportifs convaincus de dopage s'est progressivement imposée depuis une cinquantaine d'années auprès des acteurs et des institutions concernés, on peut néanmoins pointer des écarts entre, d'un côté, un discours social dominant, tourné vers l'idée qu'il faut sanctionner des méthodes et des comportements répréhensibles; et, d'un autre côté, la réalité concrète de

\footnotetext{
* Cet article est une version adaptée d'une communication orale présentée lors du congrès « Droits de l'Homme et dopage », Université de Rennes II / Conseil de l'Europe, Rennes (25 mars 2011).
}

la lutte antidopage, marquée par des ambiguïtés et des apories notables.

À l'appui de ce constat, de nombreux travaux documentent le fait que la lutte antidopage, loin d'être monolithique, relève en fait de perspectives multiculturelles. Une réalité qui atteste a minima de perceptions différenciées de l'infraction et des objectifs attachés à la sanction; mais aussi des rapports de force qui caractérisent les relations entre les principaux détenteurs d'enjeux concernés par le dopage.

Dans le détail, et selon une tendance qui semble s'être consolidée dans la dernière décennie, la lutte contre le dopage sportif dévoile une dialectique articulée autour 
de deux axes complémentaires : un axe vertical - axe $d u$ droit - construit sur la multiplication des dispositions et des dispositifs voués à la répression et à la sanction ; un axe horizontal - axe de la régulation - enraciné dans des principes d'arbitrage, de conciliation et de médiation, propres à inscrire la résolution des litiges sportifs dans des cadres attentifs aux notions de droits de l'Homme et de dignité des sportifs.

Cette gestion complexe incite alors à penser que, à côté d'un volontarisme punitif, circule également dans le système de pénalisation institutionnelle du dopage, un processus de « civilisation du droit» (Spierenburg, 1984). Il se caractérise par des aspects procéduraux tournés vers la recherche d'efficacité, le souci de rendre le droit au plus près de la situation spécifique du sport, ou encore l'opportunité de négocier les modalités de la norme et de la sanction; mais aussi par des aspects axiologiques, comme en témoignent les références régulières à des principes de droit qui entendent protéger l'individu sportif contre des empiètements et des abus en mesure de lui dénier son statut de personne porteuse de droits fondamentaux.

Le recours à la théorie du processus de civilisation du droit nous semble de bonne valeur heuristique dans la mesure où elle permet de mettre en lumière le grand nombre des variables qui organisent désormais la lutte contre le dopage : sensibilité sociale et juridique d'une époque ; judiciarisation croissante des conflits sportifs ; représentations, enjeux et intérêts distincts des acteurs concernés; fortes tensions entre les valeurs historiques morales du sport et ses valeurs modernes de performance.

Dans la présente contribution, et dans les termes d'une approche croisant la sociologie et l'analyse juridique, nous illustrerons notre propos en nous interrogeant sur le sens et les ambiguïtés du projet-pilote de lutte antidopage Windop. Ce projet, conduit à l'Institut des sciences du sport de l'Université de Lausanne depuis mars 2010, avec l'aide d'un cabinet spécialisé dans le coaching des sportifs, et soutenu financièrement par l'Agence mondiale antidopage (AMA), vise, selon ses initiateurs, à « donner aux athlètes condamnés pour des faits de dopage des outils afin de comprendre leur acte, de ne pas récidiver et de se réinsérer dans leur existence sportive » (www.unil.ch/ issul). On peut mentionner que cet article, élaboré sur un matériau (intégration documentaire, entretiens) récolté entre 2010 et 2011, ne concerne que la seule phase de mise en œuvre de Windop. De même, les commentaires formulés se réfèrent au Code mondial antidopage (CMAD) en vigueur à cette époque.

\section{Lutter contre le dopage sportif : discours dominant mais nécessité de contrepoids}

Les politiques de lutte antidopage, en dépit de périodes de flou réglementaire et de concernements parfois variables des acteurs du domaine, attestent, depuis une cinquantaine d'années, d'une extension du droit et de la pénalité, ainsi que d'une volonté de coordination croissante dans la résolution de cette préoccupation. Un souci qui culmine, après l'élaboration par l'Union européenne, en 1992, d'un code de conduite contre le dopage, dans la création, intervenue en 1999, de l'AMA et dans la formulation afférente du CMAD, entré en vigueur à l'échelon mondial en 2004 pour le mouvement sportif, et en 2006 pour les gouvernements. Progrès notable puisque cette Agence constitue le premier organisme mondial paritaire entre le mouvement sportif et les Etats en lien avec des questions touchant au domaine sportif.

Cette dynamique de régulation révèle toutefois d'importantes dissonances. On connaît, à cet égard, la régularité des conflits qui ont opposé interprétations, experts et référentiels pour réguler un phénomène qui présente quatre complexités dont il est établi qu'elles sont génératrices d'importants dissensus.

\subsection{Une première complexité : l'organisation sportive}

Sur ce point, Mignon (2002) montre clairement que la lutte contre le dopage s'inscrit dans des environnements institutionnels caractérisés par des enchevêtrements considérables. Coexistent en effet au sein de l'organisation du sport mondial, tantôt se confrontant tantôt coopérant, des fédérations nationales et internationales, des organismes faîtiers, mais aussi des États; c'est-à-dire autant de souverainetés, sportives et politiques, en lutte pour le contrôle de leurs prérogatives et le maintien de leurs puissances respectives. Dès lors, l'efficacité de la lutte contre le dopage est contingente et relative et s'exerce en fonction des stratégies des différents stakeholders.

\subsection{Une deuxième complexité : la confrontation justice sportive/justice civile}

Ici encore, la littérature met l'accent sur diverses déclinaisons d'une confrontation qui montre la régularité des heurts entre ces deux fabriques du droit. Deux de ces conflictualités sont récurrentes. En premier lieu, la question de la commensurabilité entre les règles édictées par les institutions sportives et les procédures prévues par le droit ordinaire. En second lieu, la question de la diffusion, au sein d'un monde sportif longtemps organisé autour de principes d'autorité verticaux et patriarcaux, du modèle anglo-saxon de judiciarisation, qui installe les sportifs au statut de justiciables ordinaires, engagés dans la défense d'enjeux qui leur sont propres. Cette judiciarisation a régulièrement transformé des conflits sportifs en des conflits relevant du droit du travail; les suspensions sportives étant alors assimilées à des entraves à l'activité professionnelle et la réparation du préjudice subi pouvant déboucher sur des demandes de compensations financières. 


\subsection{Une troisième complexité : la question des contrôles et de leur légitimité}

Schématiquement parlant, les politiques de lutte contre le dopage s'articulent autour de trois nœuds : la liste des substances et des pratiques interdites, les contrôles et les prélèvements biologiques et enfin, le type et la rigueur des sanctions à apporter aux infractions constatées. Il est avéré à ce propos que ces trois thèmes constituent autant de foyers de conflits : les fédérations sportives tardent à se mettre d'accord autour d'un catalogue unifié des produits interdits; le principe des tests, des procédures et de la légitimité des laboratoires sont régulièrement contestés; le régime de dévolution de la faute, la désignation du coupable et le châtiment à apporter ne sont pas stabilisés, donnant même à voir des perceptions variables. Dans ces termes, la cohérence des mesures antidopage sont alors captives de laborieuses réductions agonistiques.

\subsection{Une quatrième complexité : la démonstration scientifique de la prise de produits}

La lutte contre le dopage sportif pose comme l'un de ses principes de base la désignation de l'infraction par des validations établies par la raison biomédicale. Or, et comme on l'a montré (Pech, 2000), les preuves scientifiques du dopage, bien qu'elles soient appuyées sur des procédures et des vérifications légitimées par les canons de la science, n'échappent pas au problème plus général de la réfutabilité des énoncés. Dans ces termes, les preuves utilisées pour caractériser le dopage sont susceptibles d'être prises en défaut et ne constituent alors pas des arguments incontestables.

\section{Des dispositifs civilisateurs}

Ainsi, dans le même temps où de nombreux éléments dessinent l'évidence d'une mise en cohérence répressive du phénomène du dopage, on peut voir que cette trajectoire punitive est régulièrement freinée par des dispositifs civilisateurs qui viennent désormais marquer la forme courante de résolution des litiges sportifs, en amoindrissant la dimension contraignante, offrant même des contrepoids au substantialisme de la loi réputée peu adéquate pour contenir unilatéralement la question du dopage et, plus généralement, de l'infraction sportive.

Nous entendons par dispositifs civilisateurs des constructions institutionnelles et des maillages procéduraux de régulation, de résolution et d'arbitrage des conflits sportifs, dotés d'un objectif commun - dépasser le cadre formel du rôle de la stricte procédure en droit - et articulés sur un argumentaire dont trois énoncés au moins sont immédiatement saillants : l'exceptionnalité juridique, professionnelle et sociologique des mondes du sport (Foucher, 2007; Oswald, 2007); l'impératif du recours à des procédures souples facilitant la négociation par la communication et le dialogue (Carrard, 2007; Foucher, 2007); la nécessité de préserver la dignité des contrevenants, en leur garantissant le respect de certains droits fondamentaux menacés par les abus et les empiètements de la justice ordinaire (Oswald, 2007).

On peut illustrer l'importance de ces inflexions civilisatrices au travers de l'influence désormais exercée par des maillages juridiques - telles certaines lois nationales -, par l'action d'institutions telles que le Tribunal arbitral du sport (TAS) et l'AMA, ou encore par le poids croissant de la logique biomédicale dans la gestion du dopage. Autant de signes révélateurs d'un double phénomène : en premier lieu, que la lutte contre le dopage s'inscrit aujourd'hui à l'intérieur d'un continuum qui est celui des nouveaux droits économiques, sociaux et sanitaires; en second lieu, que l'on entend définir une nouvelle compréhension des sanctions et de l'exécution de la peine.

Le Code du sport français (art. L 230 ss), bien que rigoureux, s'articule par exemple autour d'un triangle civilisateur qui entend privilégier un strict respect de la procédure (souci méticuleux des droits de la défense et des procédures contradictoires, indépendance des instances de contrôle et organisation de recours), empêcher les atteintes à la dignité des personnes; mais aussi dépénaliser le dopage quand il est le fait des sportifs, reconnus comme des victimes d'un entourage malveillant et auxquels il convient d'apporter informations, soins et compassion.

L'action du TAS, juridiction d'arbitrage de première instance et d'appel créée le 30 juin 1984 à l'initiative du président du CIO, tend dans le même sens. Ce tribunal évoque à son tour, au sujet de l'incompréhension présumée de la justice ordinaire dans le domaine des conflits sportifs, les revendications pour des modèles alternatifs et civilisateurs censés garantir trois impératifs au moins : l'impératif d'une justice 《douce » et située, proprement humaine, proche des citoyens-sportifs et apte à régler leurs problèmes en équité (Oswald, 2007); l'impératif de restitution du litige sportif à la communauté sportive; enfin, l'impératif de décence et de dignité humaine, étant entendu que les sanctions qui frappent les sportifs mettent en cause des droits personnels fondamentaux, le droit d'exercer une profession notamment.

On peut encore relever l'accent mis, depuis une décennie et dans nombre de fédérations sportives nationales ou internationales, sur un affinement des techniques scientifiques propres à confondre des compétiteurs usagers de substances et de méthodes difficiles à détecter (dosage préventif du taux d'hématocrite, passeport biologique). Une telle volonté d'améliorer encore la récolte des preuves de violations des règlements antidopage n'est toutefois pas vierge de références civilisatrices, en l'espèce la préservation de la santé des sportifs.

Sur ce point, on peut montrer que l'axe de la vigilance biomédicale et du souci de l'intégrité de la santé des sportifs a pris le pas sur l'axe pénal et disciplinaire; les approches sanitaires et sociales du dopage venant alors se confronter aux approches «historiques », fondées sur la 
prohibition des consommations (Pech, 2000). Indice fort d'une sensibilité nouvelle qui reconnaît désormais au sportif de métier une dignité de travailleur et un droit afférent à la protection de sa santé.

\section{Le Code mondial antidopage et ses révisions : les sportifs comme des personnes}

Le CMAD, en particulier dans les révisions substantielles qu'il a connues à l'occasion de la $3^{\mathrm{e}}$ conférence mondiale sur le dopage, qui s'est tenue à Madrid au mois de novembre 2007, dévoile de notables inflexions civilisatrices, qui semblent en assouplir son orientation déclarée vers la sévérité et la cohérence punitive. Au profit de garanties générales, telles le respect des spécificités du contentieux sportif, de l'équité entre citoyens, mais aussi de l'administration d'une justice plus informée des contraintes des contextes de vie des sportifs.

Pour rappel, le CMAD a pour ambition de systématiser et d'uniformiser les principes régissant la lutte contre le dopage, ce tant pour les institutions sportives que pour les États. Son entrée en vigueur en 2004 atteste donc d'un évident changement de paradigme dans la définition du dopage. Désormais, celui-ci n'est plus « considéré comme un acte humain ou le résultat d'un tel acte, mais comme la violation des règles antidopage décrivant cet acte ou son résultat ${ }^{1} \gg$.

En 2006 toutefois, l'AMA a initié une série de révisions du Code redéfinissant de fait la question des contrôles et des sanctions dans le sens d'une sensibilité accrue aux exigences de la vie sportive et à la dignité des sportifs désormais reconnus comme des sujets singuliers. Deux réformes du CMAD semblent s'en inspirer.

La première nouveauté a trait à la modification du système de la localisation des sportifs en vue de contrôles hors compétition. Celui-ci prévoit que les sportifs qui ont été désignés par la fédération internationale à laquelle ils sont affiliés doivent indiquer au moins une heure par jour (et non plus chaque jour entier) le lieu où ils seront à disposition des contrôleurs pour être testés. Dans ce contexte, la conférence a uniformisé les critères relatifs à la qualification des infractions concernant le nombre des contrôles manqués ou la non-transmission des informations sur la localisation géographique des sportifs. L'article 2.4 CMAD modifié prévoit à présent des sanctions après trois manquements en 18 mois, alors que par le passé, le choix était laissé à la discrétion des fédérations (dont le couperet tombait en général après 2 à 3 manquements). Elle a ainsi élargi les clauses compréhensives d'oubli, de négligence ou encore de raisons pratiques.

La seconde nouveauté du Code a trait à la flexibilité des sanctions. La version antérieure du Code avait, en effet, adopté une approche qui ne laissait que très peu de discrétion quant à la suspension infligée. Celleci était automatiquement de deux ans et elle ne pouvait

\footnotetext{
1 Traduction libre de Janwillem (2003).
}

être réduite - et au maximum de la moitié seulement qu'en cas d'absence de faute ou de négligence « significative ». Dans le CMAD de 2007, l'éventail des sanctions s'est élargi, avec la possibilité de suspendre un athlète jusqu'à quatre ans en cas de circonstances aggravantes et, pour les substances spécifiées, de réduire la peine de deux ans jusqu'à une réprimande. En outre, la liste des substances spécifiées a été élargie (art. 10.2 et 10.4), les autres substances se réduisant désormais aux stéroïdes, aux hormones de croissance et à l'EPO. Enfin, le Code autorise désormais une réduction des sanctions ou un sursis partiel, non seulement en cas d'absence de faute ou de négligence significative (toujours pour la moitié au maximum), mais aussi en cas d'aide substantielle à la lutte contre le dopage ou d'aveux.

\section{Le projet Windop : enjeux et ambiguïtés d'une approche reconstructive}

Comme on l'a vu, le CMAD se concentre essentiellement sur la répression du dopage. Il n'aborde donc pas la question du suivi médical et psychologique des sportifs convaincus d'une violation des règles antidopage. La mise en œuvre récente, en Suisse, du projet Windop, soutenu financièrement par l'AMA, paraît devoir combler cette lacune en même temps qu'il semble incarner au plus près la place croissante prise par de telles enclaves civilisatrices et, plus encore, par la tendance à privilégier les avantages d'une justice « restaurative » (Dubois, 2008), soucieuse de modifier et de redéfinir le sens comme les objectifs des sanctions qui frappent les coupables.

D'un point de vue général, Windop s'articule autour de deux volets principaux. Le premier est tourné vers la réhabilitation des sportifs punis à travers l'offre de diverses mesures d'accompagnement destinées à «mentaliser » la faute, éviter les récidives et reconstruire un avenir sportif, professionnel et social. Ce programme est composé d'un suivi biologique (contrôle des paramètres sanguins), d'un suivi physiologique et physique; d'un accompagnement psychologique ouvrant à la question de la redéfinition d'un plan de carrière ; éventuellement, d' «actions d'utilité publique » (témoignages, participations à des campagnes de prévention), ceci sur une base volontaire, étant donné que l'anonymat des participants est par principe garanti.

Le second volet consiste en un programme scientifique qui, sur la base de l'exploitation d'un échantillon significatif de la population engagée dans Windop ${ }^{2}$, vise à saisir quels sont les déterminants psychosociaux des conduites dopantes dans le sport. L'accès direct aux participants permet ainsi de recueillir des données sur les conduites

\footnotetext{
2 Selon les informations recueillies, 11 compétiteurs, issus des disciplines du cyclisme, du football, de l'athlétisme, du bob et de la gymnastique étaient engagés dans le protocole fin 2010 .
} 
dopantes et d'investiguer qualitativement et quantitativement les différentes composantes de ces conduites. Les résultats de ces recherches sont clairement envisagés comme pouvant alimenter les connaissances scientifiques quant aux déterminations psychologiques, sociologiques et culturelles du dopage, et comme susceptibles d'enrichir les stratégies de prévention et de formation des jeunes sportifs et des entraîneurs.

Le programme Windop se distingue encore par deux autres caractéristiques. En premier lieu, par le fait qu'il est articulé sur des contenus moraux explicites, tels que réparation, réhabilitation, restauration des liens sportifs et sociaux; ou encore assomption, par le sportif, de sa responsabilité à l'égard de ses 《victimes». En second lieu, par le fait que le principe d'une adhésion volontaire des sportifs à une procédure contraignante pose la question d'une contrepartie propre à atténuer la sévérité de la sanction infligée ${ }^{3}$.

Dans ces termes, Windop relève alors d'un projet socio-moral à teneur civilisatrice dont on peut retenir : a) qu'il atténue la dimension fautive de la prise de produits prohibés comme le résultat d'une pratique individuelle qui conduirait à désigner le sportif comme un délinquant au profit d'une perception élargie qui met l'accent sur les racines collectives et institutionnelles d'un écart aux valeurs sportives; b) qu'il met en œuvre une dynamique qui cherche à encourager un processus d'identification compassionnelle entre les accusateurs, les accusés et le public ; c) qu'il offre, enfin, les qualités d'une enclave de réhabilitation à l'issue d'une trajectoire de pénitence scandée par les étapes de retour sur et d'exhibition de la faute.

$\mathrm{Si}$, un jour, l'engagement dans Windop devait permettre d'obtenir une éventuelle réduction de la sanction des sportifs 《repentis », ce programme pourrait s'imposer comme un dispositif juridique ambigu, inspiré $\mathrm{du}$ marchandage judiciaire de type «plea bargaining $»^{4}$.

${ }^{3}$ On rappellera qu'à ce jour, aucun panel disciplinaire ne peut imposer à un sportif de suivre ce programme. En outre, à l'heure où nous écrivons ces lignes, la question de la négociation de la sanction ne constitue pas un objectif prioritaire de Windop, encore que les entretiens menés avec certains initiants du projet montrent qu'ils y sont favorables.

4 Selon la définition donnée par le dictionnaire juridique Black's, le «plea bargaining » est «un accord négocié entre un procureur et un accusé aux termes duquel l'accusé plaide coupable pour une infraction moindre, ou pour l'un des multiples chefs d'accusation, en échange d'une concession par le procureur, habituellement d'une peine moins sévère ou d'un abandon d'autres chefs d'accusation ». On considère qu'aux États-Unis plus de $90 \%$ des affaires pénales se concluent par une condamnation sur la base d'un aveu du prévenu (Garner, 1999). Si le «plea bargaining» est généralement absent en Europe, il n'en reste pas moins que le «bargaining» («marchandage ») existe sous diverses formes, dans nos systèmes de justice pénale. En France, une procédure de comparution sur reconnaissance préalable de culpabilité, appelée communément «plaider coupable », a été instaurée par la loi du 9 mars 2004, dite « loi Perben II ».
Même si l'on se trouve ici dans le domaine disciplinaire et non pénal, on peut relever, par analogie, que l'inspiration civilisatrice de ce mécanisme (prise en compte des circonstances personnelles, adaptation à la situation spécifique du sport, rapidité de la procédure, recherche de la négociation, pragmatisme,... ) impliquerait alors aussi la mise en péril du principe de présomption d'innocence et le déni du droit à un procès équitable du fait de l'automaticité de la déclaration de culpabilité (Gilliéron, 2010).

\section{Discussion}

La lutte contre le dopage relève aujourd'hui d'orientations complexes qui rendent visible une dialectique associant haussement de la tonalité répressive et reconfiguration des politiques du châtiment dans le sens d'une attention croissante à divers traits de civilisation.

À cet égard, on peut observer l'émergence récente de critiques qui, outre qu'elles entendent démontrer l'inefficacité des réponses strictement juridiques et punitives et les vertus des approches sollicitant davantage la négociation et le dialogue, pointent les atteintes aux droits personnels fondamentaux attachées aux applications des règlements antidopage. Les contestations les plus régulièrement formulées à l'encontre du CMAD attestent ainsi de convergences sur le problème de l'atteinte à la protection de la vie privée consécutive au système de localisation des athlètes et à la soumission obligée aux tests inopinés.

Le projet Windop - bien qu'expérimental - que nous décrivons ici comme un projet expressif d'une dynamique de civilisation du droit, en regard de nombre de ses orientations (approche sociologique et médicale de la prise de produits, modification de la perception du sens et des objectifs de la sanction, aide au retour dans les communautés sociale et sportive...) semble s'inscrire de plainpied dans ce qui révèle tout à la fois une évolution des cadres cognitifs, une politique du soupçon à l'égard du réputé manque de garanties juridiques des plus récents développements de la lutte antidopage; mais aussi le degré de complexité qui préside à la gestion institutionnelle de la pratique sportive de haut-niveau. L'ambiguïté de cette initiative reste toutefois forte et il serait erroné d'y distinguer seulement un dispositif de clémence. En effet, comme indiqué déjà, si Windop semble orienté par le souci de préserver un certain nombre de droits fondamentaux, on ne peut exclure qu'il pose problème, en particulier du point de vue des garanties de procédure.

Les ressources de l'analyse juridique procurent d'évidents gains de connaissance lorsqu'elles permettent de montrer que la prédilection d'un certain nombre d'acteurs $\mathrm{du}$ sport pour les contrepoids aux normes formelles en matière de lutte contre le dopage, relèvent sans doute aussi d'un aggiornamento à des mutations plus générales du droit pénal, en particulier l'attachement au registre de la procéduralisation et l'invocation de plus en plus fréquente des droits de l'Homme (Terré, 2007). Ces ressources peuvent être utilement complétées par une analyse sociologique. 
De ce point de vue, et outre que Windop permet d'alimenter une théorie générale des institutions attentive à montrer que l'institution sportive est un champ de forces dans lequel se confrontent des représentations, des enjeux et des intérêts multiples et conflictuels, un tel projet autorise encore deux constats.

Premièrement, que la tendance à donner à l'exercice de la pénalité un caractère plus « humain » répond à la nécessité des pouvoirs sportifs d'appuyer leur autorité sur le consentement de travailleurs sportifs désormais de mieux en mieux dotés en ressources diverses (juridiques, médiatiques, politiques notamment) pour affronter l'épreuve de la justice sportive.

Deuxièmement, que la coexistence de politiques explicitement répressives et de dispositifs mixtes axés sur la négociation des sanctions permet de gérer les injonctions contradictoires qui caractérisent la société du spectacle sportif : préserver les intérêts de sportifs engagés dans des environnements faisant valoir de fortes exigences à leur encontre, en même temps que ne pas altérer la crédibilité sociale du sport, comme pratique éthique fondée sur des valeurs fortes et universelles.

\section{Bibliographie}

Buy, F., Marmayou, J.M., Poracchia, D., \& Rizzo, F. (2009). Droit du sport. Paris : Librairie générale de droit et de jurisprudence.

Carrard, F. (2007). Arbitrage vs. Médiation. In A. Rigozzi M. Bernasconi (Eds.), The proceedings before the Court of Arbitration for Sport (pp. 13-19). Zurich, Basel, Geneva : Editions Weblaw/Schulthess.
Dubois, C. (2008). Restauration et détention en Belgique : genèse de la circulaire ministérielle du 4 octobre 2000. Droit et Société, 69-70, 479-505.

Foucher, B, (2007). Le système de conciliation dans la résolution des litiges sportifs. In A. Rigozzi, \& M. Bernasconi (Eds.), The proceedings before the Court of Arbitration for Sport (pp. 19-33) Zurich, Basel, Geneva : Editions Weblaw/Schluthess.

Garner, Bryan A. (1999) Black's Law Dictionary. $7^{\mathrm{e}}$ éd., St. Paul, Minnesota (West Group), ad «plea bargain ».

Gilliéron, G. (2010). Strafbefehlsverfahren und plea bargaining als Quelle von Fehlurteilen. Thèse de doctorat en droit, Université de Zurich.

Janwillem S. (2003). The WADA World Anti-Doping Code: the road to harmonisation. The International Sports Law Journal, 2, 2 ss.

Mignon, P. (2002). Le dopage : un état des lieux sociologique. Documents du CESAMES, 10.

Oswald, D. (2007). Tribunal arbitral ou tribunal ordinaire? In A. Rigozzi, \& M. Bernasconi (éds.), The proceedings before the Court of Arbitration for Sport (pp. 3-13). Zurich, Basel, Geneva, Editions Weblaw/Schulthess.

Pech, T. (2000). Polichinelle et la règle de droit. In F. Siri, (Ed.), La fièvre du dopage. Du corps sportif à l'âme du sport (pp. 92-101). Paris : Éditions Autrement.

Spierenburg, P. (1984). The spectacle of suffering. executions and the evolution of repression: From a preindustrial metropolis to the european experience. Cambridge : Cambridge University Press.

Terré, D. (2007). Les questions morales du droit. Paris : PUF. 